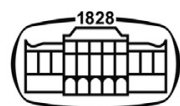

AKADÉMIAI KIADÓ

UNIVERSITY of DEBRECEN

\section{International Review of Applied Sciences and Engineering}

12 (2021) 2, 103-110

DOI:

10.1556/1848.2021.00170

(c) 2021 The Author(s)

\title{
Geometry and shape optimization of piezoelectric cantilever energy harvester using COMSOL multiphysics software
}

\author{
Ahmed A. Hashim* ${ }^{*}$, Khalil I. Mahmoud and \\ Hussein M. Ridha
}

Department of Media Technology Engineering, University of Information Technology and Communications - College of Engineering, Baghdad, Iraq

Received: August 18, 2020 • Accepted: January 23, 2021

Published online: March 4, 2021

\section{ABSTRACT}

In embedded systems that necessarily require a steady source of power and (or) attaches to a sensor(s), there are opportunities to mix small batteries to supply such power. The aim of this research is to optimize the geometry and shape of piezoelectric cantilevers to harvest more power. Several piezoelectric cantilever geometries with various shapes (rectangular, triangular, circular, and trapezoidal cross section) are tested in COMSOL multiphysics simulator to find the best geometry that provides the highest accomplishable power. The most efficient geometry was found to be conferred by the trapezoidal, cross section cantilever. Next, another improvement method was applied to maximize the harvested power of the cantilever by modifying the shape of the trapezoidal cantilever structure through increasing the number of its faces. The results demonstrated that the highest output power $(36 \mathrm{~mW})$ was produced by the four faces, trapezoidal cross section design of cantilever.

\section{KEYWORDS}

energy harvesting system, piezoelectric cantilever, geometry optimization, shape optimization, COMSOL multiphysics

\section{INTRODUCTION}

Vibration energy is among the most common and wasteful energy types in nature from which electrical energy is possibly acquired via the three ordinary processes of capacitive, electromagnetic, as well as piezoelectric harvesting. Piezoelectric materials are able to generate electrical charges upon their exposure to strains [1].

The latter harvesting mechanism is widespread due to higher strength and simplicity during application. Most of harvesters of this kind employ single- or multi-shaped cantilever beam structures in which a beam is fixed at an end of a device made of one to several layers connected to an elastic metal layer, that renders the structure more elastic and the piezoelectric material less breakable [2].

Upon application, the piezoelectric impact of a small generating device is employed for

*Corresponding author. Department of Media Technology Engineering, University of Information Technology and Communications, College of Engineering, Al-Nidhal Road, Baghdad, Iraq.

E-mail: dr.ahmed.hashim@uoitc.edu.ic the harvesting of mechanical, i.e. vibrating energy and transforming it into electrical power [3]. The efficiency of such devices can be influenced by various piezoelectric materials, e.g. lead zirconide $(\mathrm{PZT})$, zinc oxide $(\mathrm{ZnO})$ and aluminum nitrate $(\mathrm{AlN})$, which have various constants [4].

The most important energy harvesters' applications include the implantable biomedical devices. Romero et al. [5] performed a detailed survey of the power collection devices prepared for this aim, where most of vibratory energy harvesters collect energy from the human body movement; If the biomedical device implant is designed to function in the body, then the involvement of an energy harvesting feature is favorable. 
Previous investigations described three layouts of in body-functioning harvesting systems; The first employs microbial fuel cells that oxidize glucose to create energy [6], the second relies on collecting energy from the artery stretching by covering a piezoelectric film around it [7], while the third method is by piezoelectric temperament (printing) [8].

Sunithamani et al. [9] improved the functioning of a microelectromechanical system (MEMS) piezoelectric energy harvester using an optimized PZT length, to gain a higher level of voltage, through a simulation applied by a moving mesh analysis in COMSOL Multiphysics. Detailed simulation was carried out on structures having conventional and non-conventional cross sections [10]. The drawbacks of this work were firstly that it focused only on the voltage drop across the cantilever, secondly that it compared only between different geometries, and finally that it did not mention the step size used in the simulations.

Foroughi et al. [11] presented ten various percentages of width (the fixed edge) to length (the free edge) of a rectangular cantilever, as well as two structures of exponential cantilever, all of which with constant areas that were tested using COMSOL Multiphysics. The eigenfrequencies and shapes of various cases were obtained and the plots for output voltage against frequency were achieved for the twelve modes mentioned above. The study proved that, for a rectangular geometry, the percentage of 1.2 has the highest output voltage due to its highest stress applied. The next highest voltage produced, which was at a smaller frequency of about 6 times lower than the frequency for the percentage of 1.2, was of a percentage of 0.2 , which represented $92 \%$ of the largest value. The percentages higher than 1.2 had a low output voltage ( $10 \%$ of the largest value at best) at higher frequencies. For the two exponential geometries, the voltage created was relatively lower than the largest value, reaching only $30 \%$ in the case of wide fixed edge and narrow free edge and $3 \%$ in the case of narrow fixed edge and wider free edge. Thus, it is clear that the percentage of 0.2 for rectangular geometry should be selected for energy harvesting purposes because it requires smaller available frequency and has a relatively large output voltage [12].

Aini et al. [13] and Sunithamani et al. [14] described a MEMS dependent energy harvester where a nonconventional geometry was designed so that it contains a triangletipped, T-shaped cantilever. COMSOL simulation findings revealed that the novel design was able to improve the strain distribution and produce higher voltage levels as compared to the triangular and rectangular designs at the resonance frequency. However, that work as well as the one by Williams et al. [15] focused only on the stain distribution and voltage generated, regardless of the power harvested, which is more important. Also, the author did not describe the step size utilized, which normally affects the accuracy of results. In addition, the study considered only geometry change while neglected the shape change.

Goldschmidtboeing et al. [16] analyzed various beam shapes of the piezoelectric harvesters, with a concept that depended on the well-recognized Rayleigh-Ritz approach of piezoelectric compound structures. The authors proved the superiority of the triangular-over the rectangular-shaped beams with respect to the homogeneity of the curvature regardless of the proof mass. Such a result could be reversed through the adverting mass distribution as well as rendering the triangular-shaped beams more stiff. Thus, only a slight impact was imposed by beam shape upon the overall performance. However, the triangular-shaped beams were basically of higher efficiency as compared to the rectangular ones as related to the adjustable excitation amplitude along with the higher output power. The drawback of this study lies in the fact that it considered only the shapes of the cantilever, whereas the geometry and the step size were not considered.

Dietl et al. [17] suggested a new method of altering the shape of the beam to maximize the strain in some of its sections where most of the transduction is contributed. Beams were modeled with various shapes and tested across a wide band, including the lowest two cases of vibration. The best beam shape was computed utilizing a heuristic optimization code and its characteristics were explained. Next, beam shapes were improved to permit large base excitation (constrained by highest root strain). Lastly, the ratio of endmass to beam-mass was investigated parametrically, relating increased transduction with large beam mass [18]. This study, however, dealt with the relation of output power to beam mass without considering geometry and shape relations to the harvested power.

Clearly, the previous attempts to achieve maximum energy gain were hindered by drawbacks related to the exclusion of certain essential parameters such as geometry and shape of the piezoelectric device, energy as an output parameter, and the step size applied when running COMSOL. In this work, COMSOL simulation with optimal step size was applied to optimize the geometry of the piezoelectric layer of four various shapes, three of which belonging to the rectangular cantilever (with rectangular, triangular, or cylindrical cross sections) and one is for a trapezoidal crosssectioned cantilever. Then, within the optimized geometry, different sub-geometries of the trapezoidal cross-sectioned cantilever were optimized by changing number of faces via simulation with the same software. Several output parameters of maximum performance (voltage, power, and current) were measured and comparisons among the outcomes were made.

This paper is organized as follows: the first section includes the literature survey, the second section is the methodology applied, the third section describes COMSOL Multiphysics implementation, and, finally, the fourth section presents the conclusions.

\section{METHODOLOGY}

\subsection{Mathematical basis of cantilevers}

Cantilever is any structure that has a fixed part, on one side, and a free part on the other side. Cantilever with elastic 
metal provides higher elasticity and is stronger by virtue of the elastic metal layer and hence is considered as a good application for piezoelectric devices. Herein the theoretical basis of cantilevers is provided and adopted for the calculations of various output parameters used, mainly current (and hence energy) and resonance frequency.

Stoney's equation below is utilized to compute the resonance frequency of a basic cantilever beam while it is exposed to an external force.

$$
\delta=\frac{3 \sigma(1-v)}{E\left(\frac{L}{t}\right)^{2}},
$$

where $\delta$ is the deflection of cantilever's end, $\sigma$ is the amount of force used for it, $v$ is the ratio of Poisson, $E$ is Young's Modulus, $L$ is Cantilever length, and $\mathrm{t}$ is Cantilever thickness. Cantilever spring constant $k$ is extracted by utilizing the next equation:

$$
k=\frac{3 E I}{L^{3}}
$$

where $I$ is moment of inertia of the cantilever, the equation of which is as follows:

$$
I=\frac{b h^{3}}{12}
$$

where $b$ is the bar width and $h$ is the depth. Definition of the resonance frequency can be achieved by:

$$
f_{(\text {res. })}=\frac{0.32 \sqrt{k}}{\sqrt{m}},
$$

where the mass $m=\rho h L w$, where $\rho$ is the resistivity, $h$ is the cantilever's depth, $L$ is the cantilever's long and $w$ is the cantilever's width. The equation implies that resonance frequency is dependent on spring constant and cantilever's mass.

\subsection{Experimental approach}

Piezoelectric energy harvester with various shapes and arrangement was prepared and simulated using COMSOL Multiphysics 4.5. The 3D objects were prepared utilizing Google sketch up 2019 and imported to COMSOL Multiphysics.

COMSOL ensures a very good control over the model and dimensions of the $3 \mathrm{D}$ object. It also enables testing multiple designs in a very short time, as the Google sketch up is very easy to learn and very efficient in producing 3D objects. Fig. 1 presents a flowchart that describes the steps of work within COMSOL Multiphysics.

The following steps were applied to select the most suitable properties, with the decision being made after applying multiple runs within the software:

- Type of material: Many types of piezoelectric materials were tested for various parameters and showed similar patterns of behavior, i.e. similar inverse or direct proportionality towards the specific parameters. Among these, the (PZT-5A) was selected for further analysis since

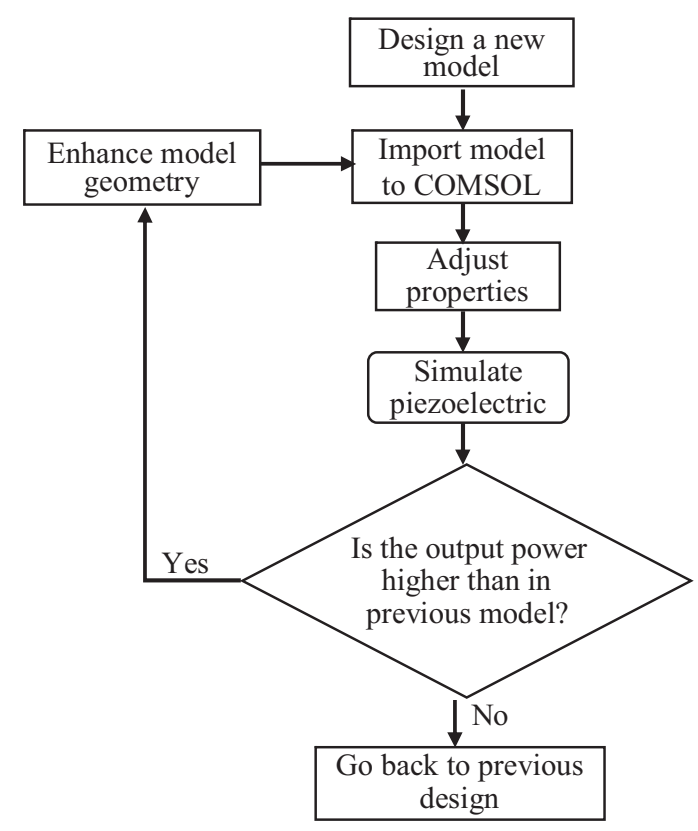

Fig. 1. Flowchart shows COMSOL simulator used for geometry optimization for maximum energy harvesting

it is common and widely utilized in similar applications, although PMN-PT and PZN-PT shows higher efficiency in harvesting energy but they are rarely used due to high cost.

- Size of device: Various sizes of the device were tested and the results showed that size (mostly length) is directly proportional with the generated energy and reversely proportional with the current. Accordingly, one size was selected for further analysis.

- Shape of device: Several shapes of the device, e.g. A, E, F, and I shapes, were tested with almost the same length. The results showed that the I shape of the device generated the highest level of energy.

- Dimensions of the selected device shape: For the selected I-shaped device, changing the dimensions via different combinations of values showed a high direct proportional correlation between energy and length, followed by width, whereas an inverse correlation was found with height.

Following the running of thousands of tests for different dimensional combinations, in which two dimensions were fixed whereas the third was considered as a variable, the effects on energy gain were examined. The results showed that the highest energy gain (and resonance for the selected material) can be achieved through the following relation, which was reached through experimental calculation rather than mathematical derivation:

$$
l-c(w / h)=0
$$

where $l$ is length, $w$ is width, $h$ is height, and $c$ is constant refered to magnitude of change.

The above relation implies that any change by one unit of width is reflected in changes in one unit of height and $c$ units 
of length. For the type of piezoelectric material used in this study, the value of $c$ was found to be 7 . Thus, the ratio of changing values is fixed, according to the relation above, to achieve the highest gain of energy and resonance frequency.

- Number of faces of the selected shape: After fixing the values of dimensions of the I-shaped device, a series of runs was conducted to optimize the number of faces (from 3 to 7 faces) for this device. The results indicated that, when a fixed power was applied, the I-shaped device with 5 faces produced the maximum gain of energy, after which any increase in the number of faces showed only slight increase in energy production.Importantly, this study compared the results based on the gain in energy rather than current, since an increase in energy does not necessarily imply increased current when the resistance of the material used is high.

Fig. 1 below shows briefly the main flow chart of the geometry design of piezoelectric material including the above criteria taking into consideration the importance of choosing step size in any simulation software [19].

\section{RESULTS AND DISCUSSION}

Fig. 2 describes a 3D unit of the piezoelectric device. The aim is to investigate the resulting distortion, pressure, and distribution of Power Harvesting Efficiency (PHE) harvesters in comparison with different shapes. The first design is a simple tapered, single section, rectangular cantilever (Fig. 2), which is $14 \mathrm{~mm}$ long and 2 and $1 \mathrm{~mm}$ wide for the wide and narrow ends, respectively.

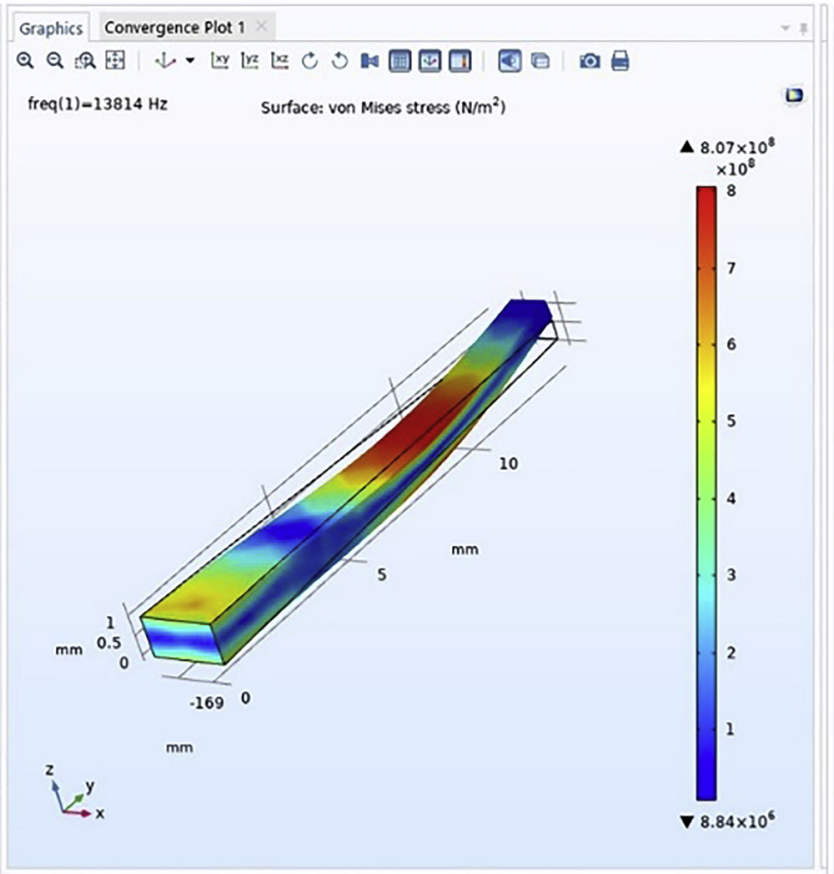

(a)
The voltage $(\mathrm{V})$ produced across an $\mathrm{R} 1=12 \mathrm{~K} \Omega$, current $(\mathrm{mA})$, and power $(\mathrm{mW})$ are demonstrated in Fig. 1, where we can observe that the resonance frequency of this design is about $13.814 \mathrm{KHz}$. The resulting peak power of this device is about $17.8 \mathrm{~mW}$ at the resonance frequency. The Second design is a simple tapered, single section, trapezoidal, three faces cantilever (Fig. 3), which is $14 \mathrm{~mm}$ long and 2 and $1 \mathrm{~mm}$ wide for the wide and narrow ends, respectively.

The voltage produced across an $\mathrm{R} 1=12 \mathrm{~K} \Omega$, current, and power are illustrated in Fig. 3, where it is clear that the resonance frequency of this design is about $2.7898 \mathrm{KHz}$. The resulting peak power of this device is about $0.42 \mathrm{~mW}$ at the resonance frequency. The third design is a triangular, single section, trapezoidal cantilever (Fig. 4), which is $14 \mathrm{~mm}$ long and 2 and $1 \mathrm{~mm}$ wide for the wide and narrow ends, respectively.

The voltage produced across an $\mathrm{R} 1=12 \mathrm{~K} \Omega$, current, and power are illustrated in Fig. $4 \mathrm{~b}$, where the resonance frequency of this design is about $2.7435 \mathrm{KHz}$. The resulting peak power of this device is about $0.05 \mathrm{~mW}$ at the resonance frequency. The fourth design is a circular cantilever (Fig. 5), which is $14 \mathrm{~mm}$ long and 2 and $1 \mathrm{~mm}$ wide for the wide and narrow ends, respectively.

The voltage produced across an $\mathrm{R} 1=12 \mathrm{~K} \Omega$, current and power are illustrated in Fig. 5b, from which we can observe that the resonance frequency of this design is about $2.8956 \mathrm{KHz}$. The resulting peak power of this device is about $0.08 \mathrm{~mW}$ at the resonance frequency. We conclude from the results above that the trapezoidal design was able to produce the maximum power when compared with power values produced by the rectangular, triangular and circular shapes.

In the following step, we considered different trapezoidal geometries by increasing the number of faces to find the best
Graphics Convergence Plot 1

Q Q Q 娄毕回回目

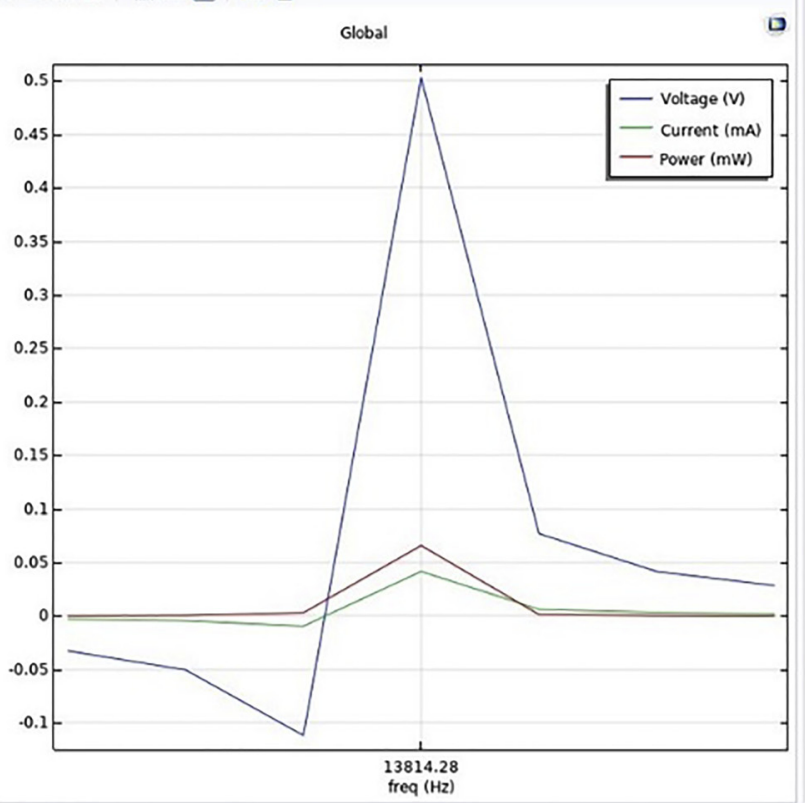

(b)

Fig. 2. Tapered single section rectangular cantilever with $14 \mathrm{~mm}$ long and 2 and $1 \mathrm{~mm}$ wide for the wide and narrow ends and $\mathrm{R} 1=12 \mathrm{~K} \Omega$ 


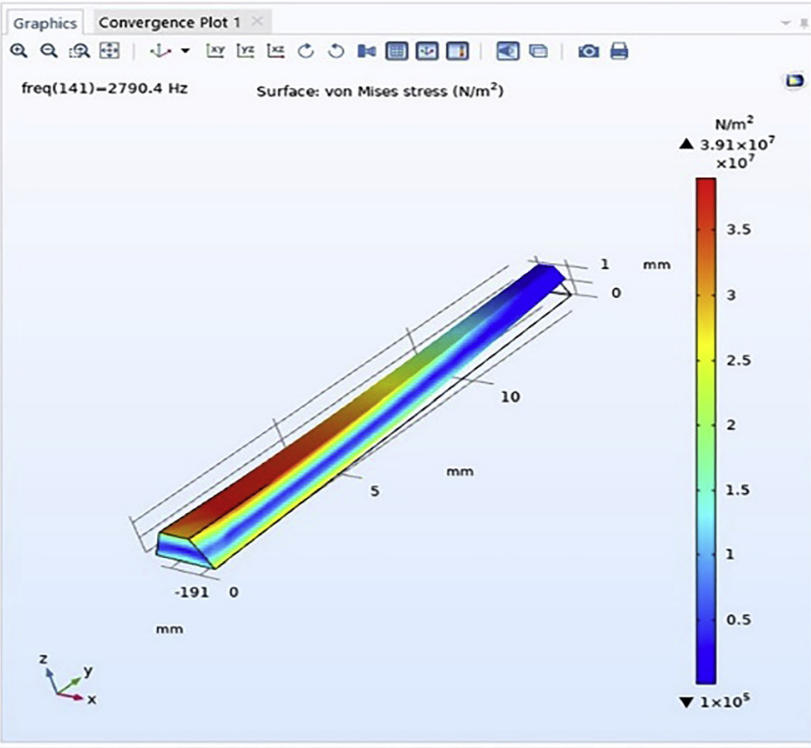

(a)
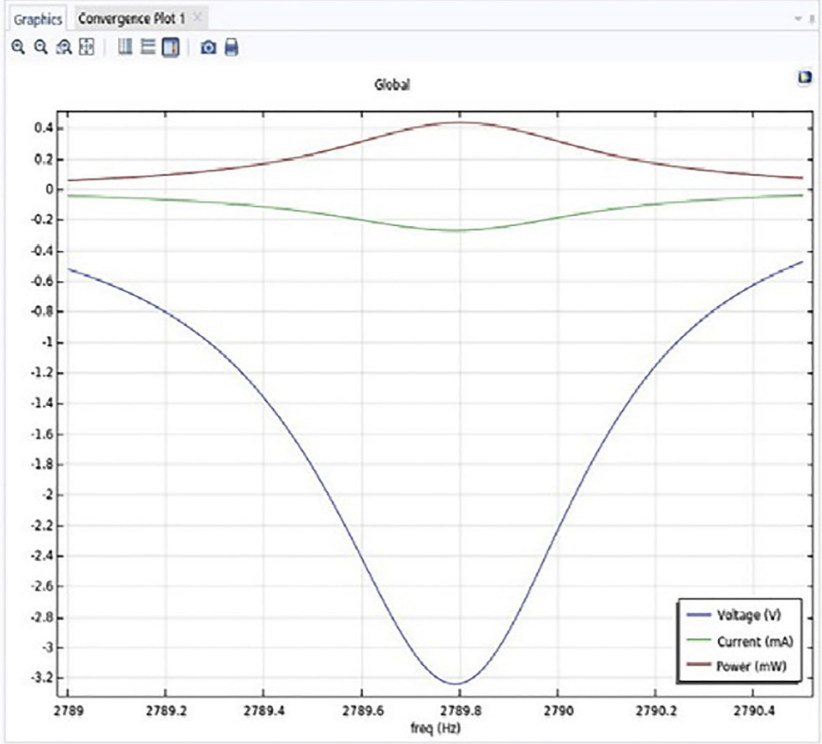

(b)

Fig. 3. Tapered, single section, trapezoidal, three faces cantilever with $14 \mathrm{~mm}$ long and 2 and $1 \mathrm{~mm}$ wide for the wide and narrow ends and $\mathrm{R} 1=12 \mathrm{~K} \Omega$

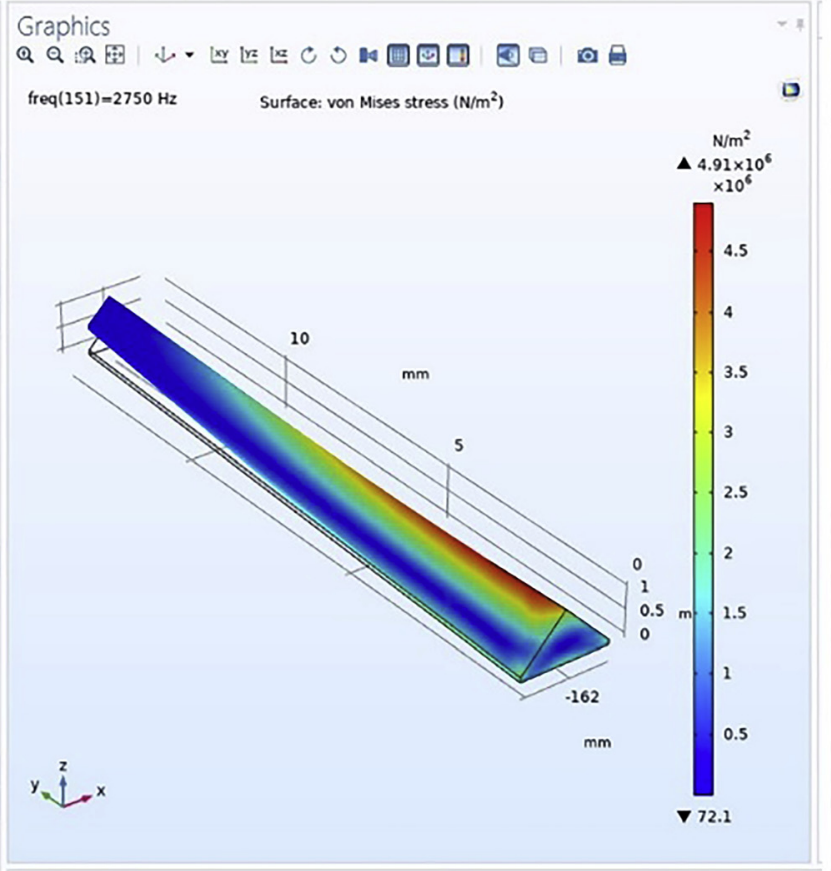

(a)
Graphics Convergence Plot $1 \times$

Q Q :

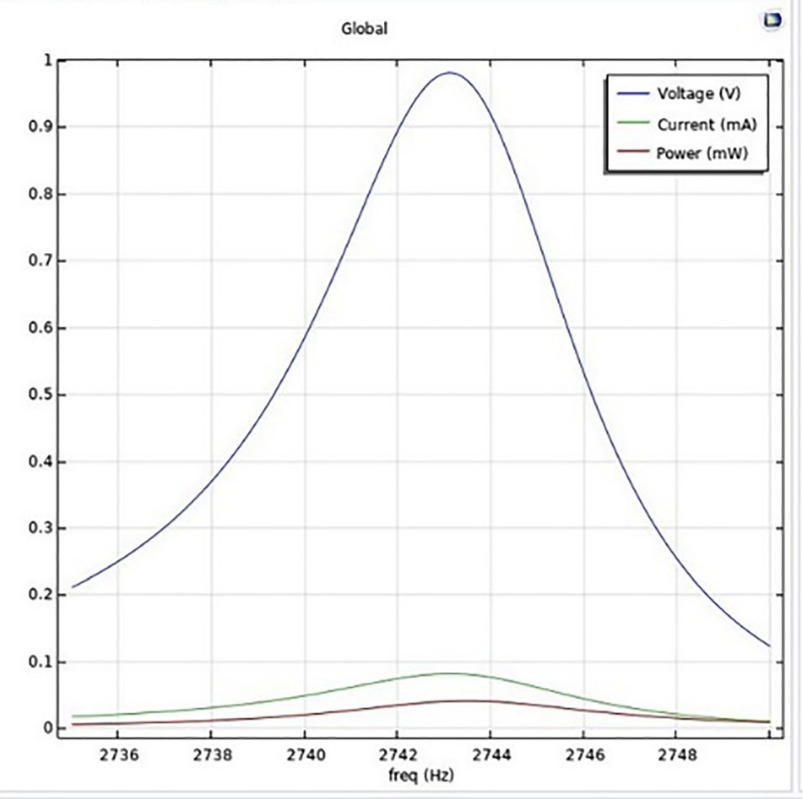

(b)

Fig. 4. Triangle, single section, trapezoidal, cantilever with $14 \mathrm{~mm}$ long and 2 and $1 \mathrm{~mm}$ wide for the wide and narrow ends and $\mathrm{R} 1=12 \mathrm{~K} \Omega$

geometry that confers the highest power harvested per single cantilever. The fifth design is a four faces cantilever (Fig. 6), which is $14 \mathrm{~mm}$ long and 2 and $1 \mathrm{~mm}$ wide for the wide and narrow ends, respectively.

The voltage produced across an $\mathrm{R} 1=12 \mathrm{~K} \Omega$, current and power are depicted in Fig. $6 \mathrm{~b}$, which demonstrates that the resonance frequency of this design is about $2.851 \mathrm{KHz}$.
The resulting peak power of this device is about $35 \mathrm{~mW}$ at the resonance frequency. The sixth design is a five faces cantilever (Fig. 7), which is $14 \mathrm{~mm}$ long and 2 and $1 \mathrm{~mm}$ wide for the wide and narrow ends, respectively.

The voltage produced across an $\mathrm{R} 1=12 \mathrm{~K} \Omega$, current and power are depicted in Fig. 7, where the resonance frequency of this design is about $2.7858 \mathrm{KHz}$. The seventh 


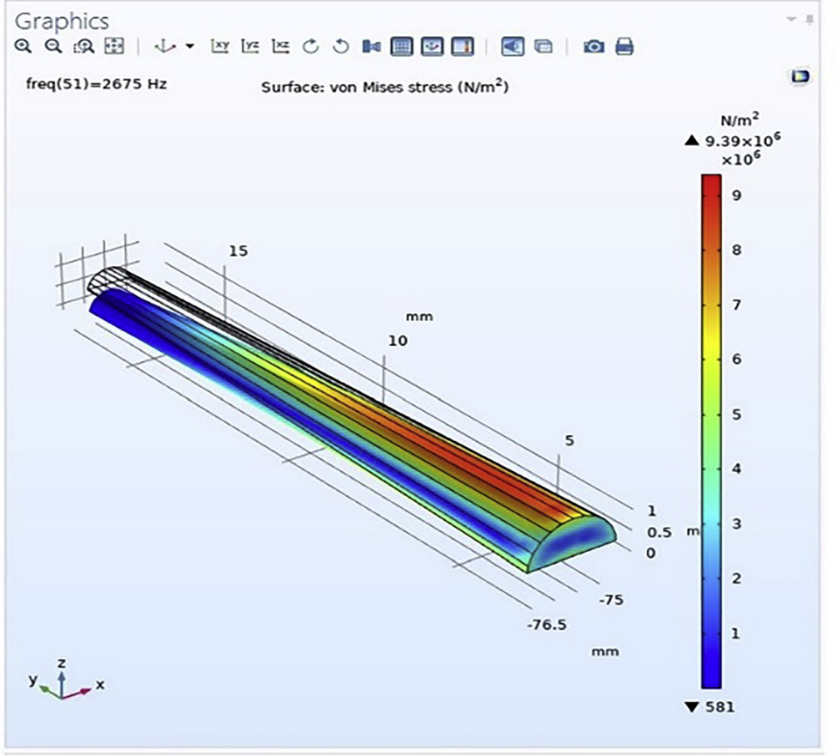

(a)

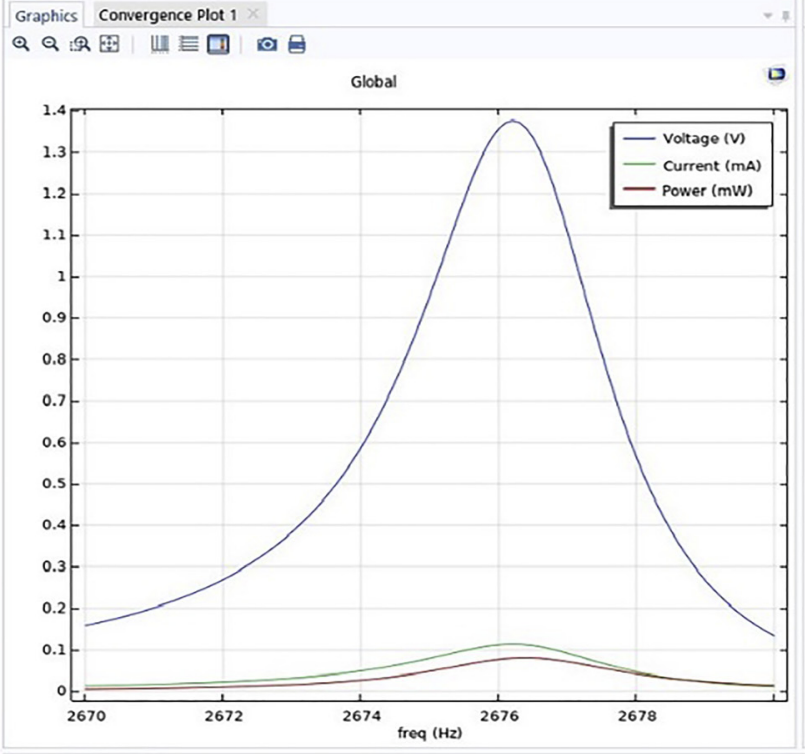

(b)

Fig. 5. Circular cantilever with $14 \mathrm{~mm}$ long and 2 and $1 \mathrm{~mm}$ wide for the wide and narrow ends and $\mathrm{R} 1=12 \mathrm{~K} \Omega$

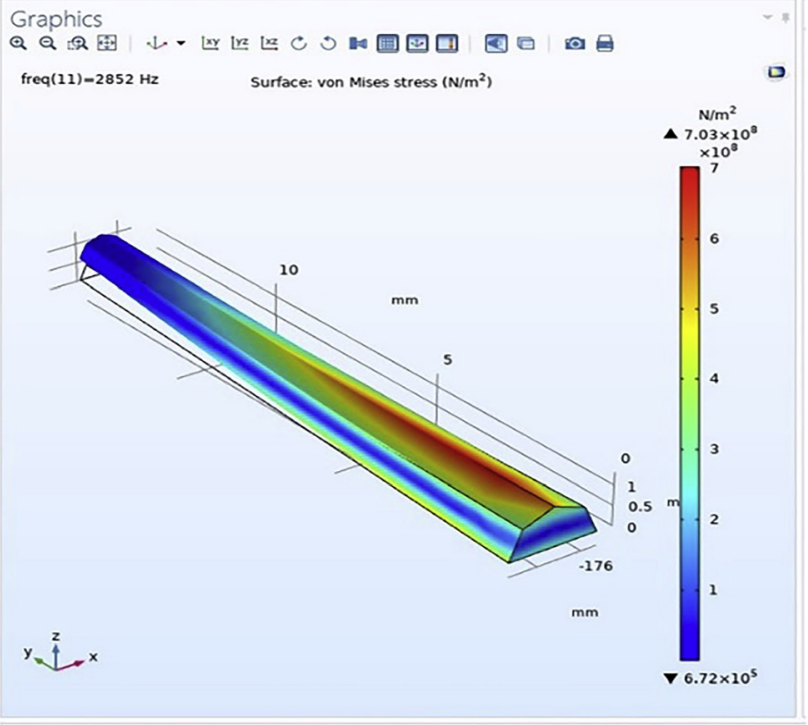

(a)

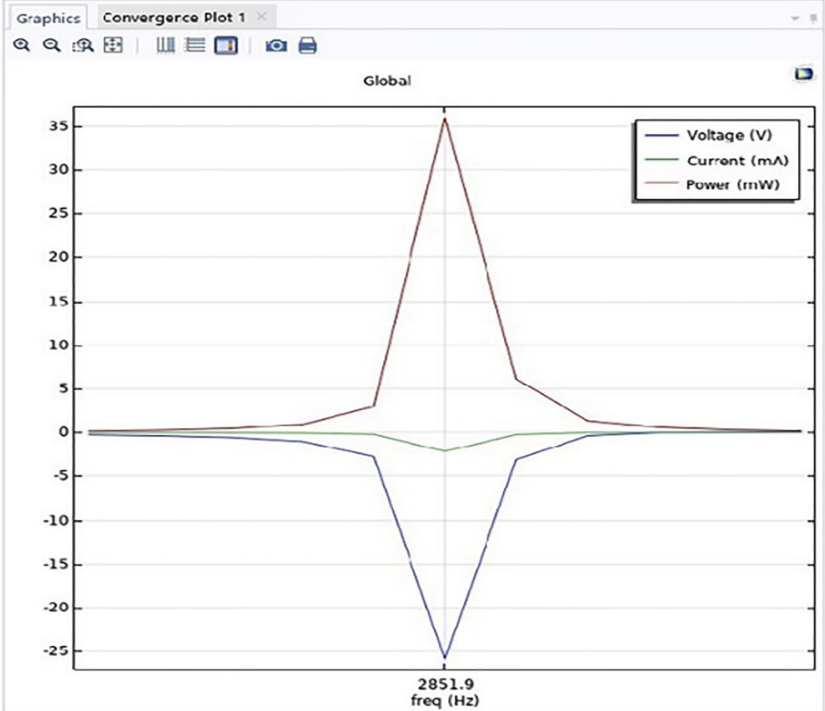

(b)

Fig. 6. Tapered single section trapezoidal four faces cantilever with $14 \mathrm{~mm}$ long and 2 and $1 \mathrm{~mm}$ wide for the wide and narrow ends and R1 $=12 \mathrm{~K} \Omega$

design is a six faces cantilever (Fig. 8), which is $14 \mathrm{~mm}$ long and 2 and $1 \mathrm{~mm}$ wide for the wide and narrow ends, respectively. The resulting peak power of this device is about $1.4 \mathrm{~mW}$ at the resonance frequency.

The voltage produced across an $\mathrm{R} 1=12 \mathrm{~K} \Omega$, current and power are demonstrated in Fig. 8, where the resonance frequency of this design is about $12.1041 \mathrm{KHz}$. The resulting peak power of this device is about $0.1 \mathrm{~mW}$ at the resonance frequency.

For detailed comparisons among the four different geometries (Rectangular, Trapezoidal 3 faces, Triangular, and Circular cross section), the Figure of Merit (FOM) is tabulated in Table 1, where the detailed performance is described including the voltage drop developed across the cantilever, the harvested power, and finally the current generated for each geometry considered in the study.

It is clear from Table 1 that the tapered, single section, trapezoidal, three faces cantilever harvested the highest output power, produced the largest voltage, and generated the maximum current among the four different shapes of cantilever. 


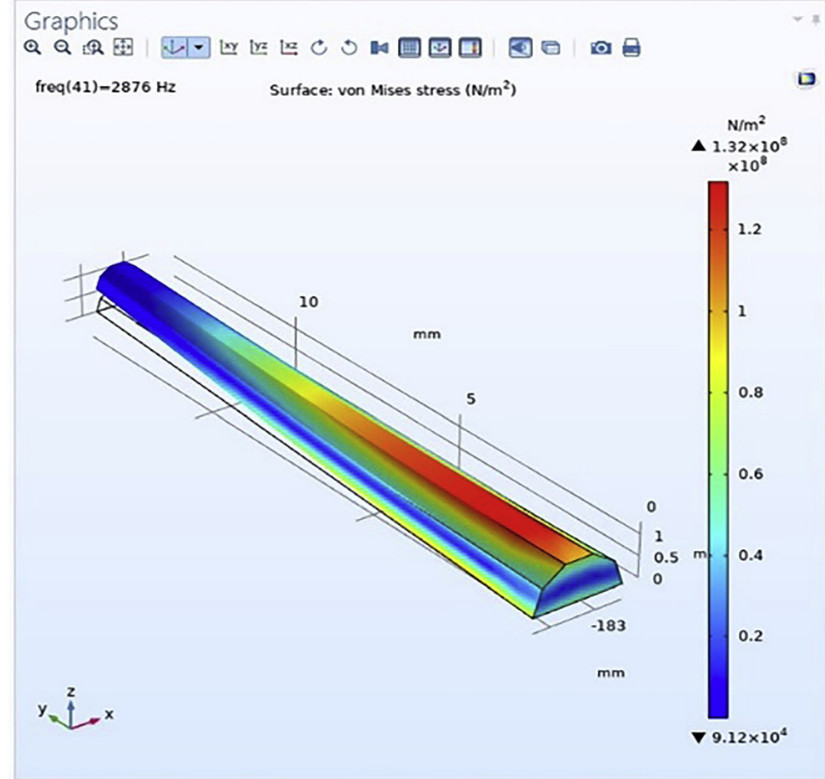

(a)
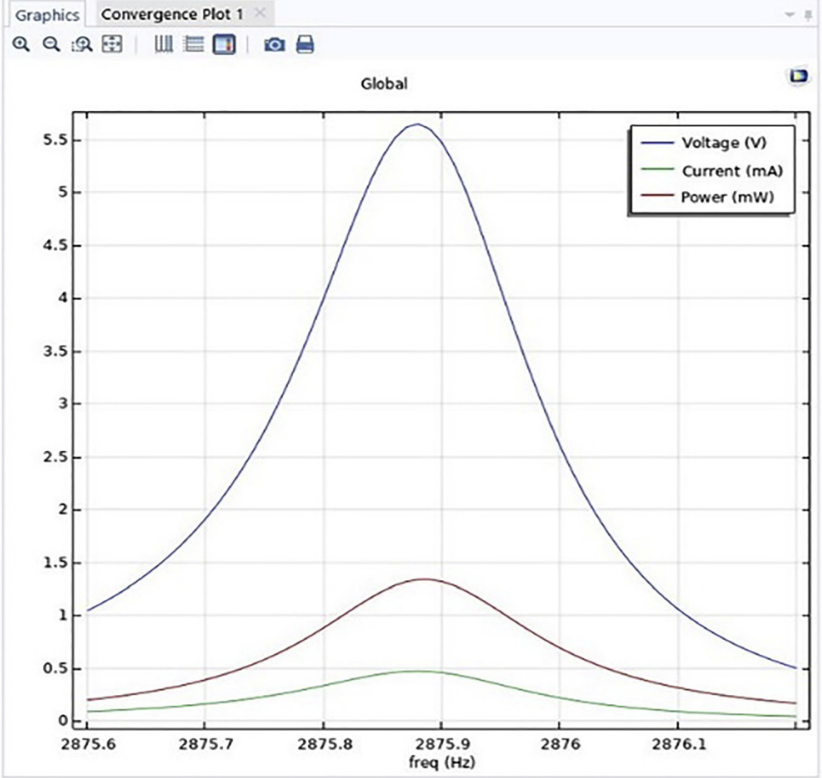

(b)

Fig. 7. Tapered, single section, trapezoidal, five faces cantilever with $14 \mathrm{~mm}$ long and 2 and $1 \mathrm{~mm}$ wide for the wide and narrow ends and R1 $=12 \mathrm{~K} \Omega$

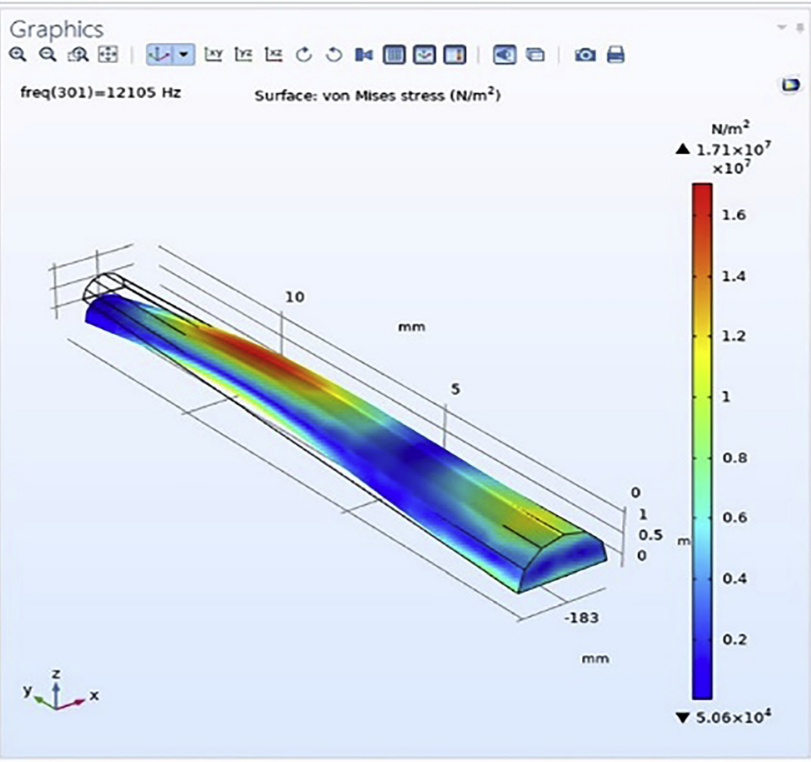

(a)

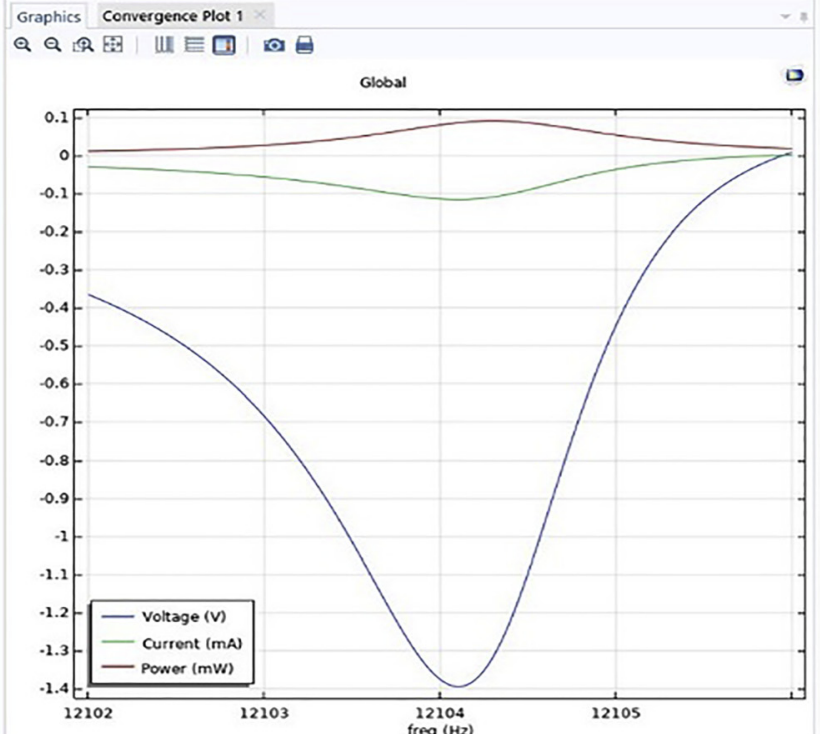

(b)

Fig. 8. Tapered, single section, trapezoidal, six faces cantilever with $14 \mathrm{~mm}$ long and 2 and $1 \mathrm{~mm}$ wide for the wide and narrow ends and R1 $=12 \mathrm{~K} \Omega$

Moreover, the comparison between the different shapes of the tapered, single sectioned, trapezoidal cantilever show that four-faces cantilever is the most efficient, given the fact that it could produce the highest output power of $36 \mathrm{~mW}$, a voltage drop of the order $-26 \mathrm{~V}$, and a generated current of $-2 \mathrm{~mA}$.

\section{CONCLUSION}

The methodology adopted in this work differs from those described in previously published works on the geometry optimization in three basics aspects, as follows: 
Table 1. Detailed performance of different geometries and shapes

\begin{tabular}{lccccc}
\hline Shape & Peak voltage $(\mathrm{V})$ & RMS power $(\mathrm{mW})$ & Peak current $(\mathrm{mA})$ & Resonant frequency $(\mathrm{kHz})$ & Step size $(\mathrm{Hz})$ \\
\hline Rectangular & 0.5 & 0.07 & 0.04 & 13.814 & 5 \\
Trapezoidal 3 faces & -3.2 & 0.42 & -0.25 & 2.8519 & 0.2 \\
Triangular & 0.98 & 0.05 & 0.085 & 2.7425 & 2 \\
Circular & 1.38 & 0.09 & 0.12 & 2.676 & 2.789 \\
Trapezoidal 4 faces & -26 & 36 & -2 & 2.8759 & 0.2 \\
Trapezoidal 5 faces & 5.6 & 1.4 & 0.5 & 12.104 & 0.1 \\
Trapezoidal 6 faces & -1.4 & 0.09 & -0.11 & & 1 \\
\hline
\end{tabular}

First, the previous studies considered only the geometry optimization, i.e. only the structure of the cantilever. While in our method, in addition to that, the best geometry was adopted and varying the shapes was considered through changing the number of faces to achieve the highest performance.

Second, the previous studies focused only on the voltage drop across the cantilevers to compare between the different geometries, our procedure concentrates on the voltage, power harvested, and the current generated upon comparison among different geometries.

Third, the most important aspect is that the step size of the resonant frequency adopted in our simulation was adopted in a very small value to ensure that the output results are accurate and can be smoothly changed and convergent to the correct values. Nevertheless, the other relative works did not mention clearly the step size used, which may lead the results to diverge or converge to inaccurate values.

\section{REFERENCES}

[1] R. S. Bindu and M. P. Kushal, "Study of piezoelectric cantilever energy harvesters," Int. J. Innov. Res. Dev., vol. 3, no. 2, pp 39-42, Feb. 2014, ISSN 2278 - 0211 (online).

[2] D. Poria, Monika, R. Sharma, D. Rohilla, and M. kumar, "Modeling and simulation of vibration energy harvesting of MEMS device based on epitaxial piezoelectric thin film," Int. J. Adv. Res. Comput. Sci. Softw. Eng., pp. 20-5, Oct. 2012.

[3] S. Ying-Jun, W. Shangguang, L. Man, G. Yang, L. Haoxiang, H. Fei, et al. "Vehicle sensor self-powered technology research based on piezoelectric," Indonesian J. Electr. Eng. Comput. Sci., vol. 15, no 3, pp. 452-7, Sep. 2015.

[4] U. Jamain, N. Hidayah, and R Ab Rahim, "Performance analysis of zinc oxide piezoelectric MEMS energy harvester," in IEEE International Conference on Semiconductor Electronics, Proceedings, ICSE, 2014, pp. 263-6.

[5] E. Romero, R. Warrington, and M. Neuman, "Energy scavenging sources for biomedical sensors," Physiol. Meas., vol. 30, no. 9, 2009.

[6] A. E. Franks, and K. P. Nevin, "Microbial fuel cells, a current review," Energies, vol. 3, no. 5, pp. 899-919, Apr. 2010.

[7] J. A. Potkay and K. Brooks, The 2nd International Conference on Bioinformatics and Biomedical Engineering, 2008, ICBBE 2008, 1580.1591, 2008.
[8] Y. Qi, N. T. Jafferis, K. Lyons, et al. "Piezoelectric ribbons printed onto rubber for flexible energy conversion," Nano Lett., vol. 10, no. 2, pp. 524-8, 2010. https://doi.org/10.1021/nl903377u.

[9] S. Sunithamani, P. Lakshmi, and E. Eba Flora, "PZT length optimization of MEMS piezoelectric energy harvester with a nontraditional cross section: Simulation study," Microsyst. Technol., vol. 20, pp. 2165-71, 2014. https://doi.org/10.1007/s00542-0131920-y.

[10] N. N. H. Ching, H. Y. Wong, W. J. Li, P. H. W. Leong, and Z. Wen, "A laser-micromachined multi-modal resonating power transducer for wireless sensing systems," Sens. Actuators A, vols. 97-98, pp. 685-90, 2002.

[11] J. Foroughi, "Geometry optimization of bimorph piezoelectric cantilever energy harvester for maximum output voltage," in The 8th International Conference on Acoustics \& Vibration, ISAV, 2018.

[12] S. Chalasani and J. M. Conrad, "A survey of energy harvesting sources for embedded systems," in IEEE SoutheastCon, 2008.

[13] A. Aini, A. N. Nordin, and H. Salleh, "A comparative study on MEMS piezoelectric microgenerators," Microsyst. Technol., vol. 16, pp. 1673-81, May 2010. https://doi.org/10.1007/s00542-010-10869.

[14] S. Sunithamani, P. Lakshmi, and E. Eba Flora, "Simulation and optimization of MEMS piezoelectric energy harvester with a nontraditional geometry," in The Proceedings of the 2012 COMSOL Conference, 2012.

[15] C. B. Williams and R. B. Yates, "Analysis of a micro-electric generator for Microsystems," Sensors and Actuators A, vol. 52, pp. 8-11, 1996.

[16] F. Goldschmidtboeing and P. Woias, "Characterization of different beam shapes for piezoelectric energy harvesting," $J$. Micromech. Microeng., vol. 18, no. 10, pp. 1-7, 2008. https://doi. org/10.1088/0960-1317/18/10/104013.

[17] J. M. Dietl and E. Garcia, "Beam shape optimization for power harvesting," J. Intell. Mater. Syst. Struct., vol. 21, no. 6, pp. 633-46, March 2010. https://doi.org/10.1177/1045389X10365094.

[18] A. M. Mohd Asry, F. Mustafad Asry, M. Ishak, and A. Ahmad, "Power generation by using piezoelectric transducer with bending mechanism support," Int. J. Power Electron. Drive Syst. (IJPEDS), vol. 10, no. 1, pp. 562-7, Mar. 2019.

[19] M. M. Rasheed, A. K. Faieq, and A. A. Hashim, "Android botnet detection using machine learning," Ingenierie des Systemes d'Information, vol. 2, no. 1, pp. 127-30, 2020. 